\title{
Espiritualidade e religiosidade como estratégia de enfrentamento do câncer de mama: relato de um grupo de pacientes
}

\author{
Cléria Maria Bittar* \\ Reivane Lopes Cassiano** \\ Laura Nascimento Silva***
}

\begin{abstract}
Resumo
Este estudo teve a finalidade de conhecer o papel da espiritualidade no enfrentamento do tratamento de pacientes que sofreram mastectomia. A pesquisa foi realizada na Clínica Escola de Fisioterapia de uma universidade particular de um município paulista. Participaram da pesquisa cinco mulheres com idade entre 48 e 79 anos que estavam em processo de reabilitação. As entrevistas tinham o intuito de conhecer o papel da religiosidade/espiritualidade no enfrentamento da enfermidade, com duas categorias centrais: reação inicial ao diagnóstico e enfrentamento da enfermidade, e a espiritualidade como recurso no enfrentamento da doença. Algumas participantes relataram ter recebido o diagnóstico de maneira tranquila e outras de maneira muito desfavorável, sendo que a espiritualidade em ambos os casos, foi imprescindível para o enfrentamento do adoecimento e para a recuperação da saúde. A maioria relatou ter uma religião antes do diagnóstico, mas que a participação na vida religiosa/espiritual, aumentou após o diagnóstico recebido. Elas disseram da importância de serem escutadas pelos profissionais da área da saúde, pois se sentiram ajudadas. Conclui-se que a espiritualidade teve um papel fundamental no processo de enfrentamento do adoecimento proporcionando às participantes a possibilidade de alcançar bem-estar e a capacidade de continuar lutando contra a enfermidade e a favor da vida.
\end{abstract}

Palavras-chave: espiritualidade; saúde; mastectomia, enfrentamento.

\section{Spirituality and Religiosity as a strategy for coping breast cancer: Reports of a group of patients.}

\begin{abstract}
This study aimed to know the role of spirituality in coping with the treatment of patients who underwent mastectomy. The research was conducted at the Clinical School of Physical Therapy of a private university in a city of São Paulo. Five women aged between 48 and 79 years, who were in the process of rehabilitation, participated in the study. The interviews aimed to understand the role of religiosity / spirituality in coping with the disease, with two central categories: initial reaction to the diagnosis and coping with the disease and spirituality as a resource in coping with the disease. Some participants reported having received the diagnosis in a calm and other way in a very unfavorable way, and spirituality in both cases was essential for coping with illness and recovery of health. Most reported having a religion prior to diagnosis but that participation in religious / spiritual life increased after the diagnosis received. They said of the importance of being listened to by the health professionals, because they felt helped. It is concluded that spirituality played a fundamental role in the process of coping with illness by providing participants with the possibility of achieving well-being and the ability to continue fighting disease and for life. It is concluded that spirituality played a fundamental role in the process of coping with illness by providing participants with the possibility of achieving well-being and the ability to continue fighting disease and for life
\end{abstract}

Keywords: spirituality; health; mastectomy; coping.

\footnotetext{
* Docente do curso de Psicologia e do PPG Mestrado e Doutorado em Promoção da Saúde da Universidade de Franca.

** Psicóloga Centro Paula-Souza - Universidade de Franca

***Psicóloga Clínica - Universidade de Franca
} 


\section{Introdução}

O câncer de mama é o segundo tipo de câncer mais frequente no mundo entre as mulheres, com cerca de $28 \%$ novos a cada ano. Seu prognóstico é bom se diagnosticado e tratado de forma correta. Entretanto no Brasil, as taxas de mortalidade por câncer de mama ainda continuam elevadas, o que leva a se concluir que a doença ainda é diagnosticada em estágios mais avançados (INCA, 2017).

Apesar dos grandes avanços nos estudos e tratamentos do câncer, a enfermidade é ligada às profundas transformações corporais e psíquicas, bem como ter que lidar com a possibilidade da finitude da vida, o que acarreta sofrimento psíquico (Botelho, \& Pereira, 2015).

Os efeitos psicológicos e emocionais abalam a autoimagem, a sexualidade e autoestima. Pois, ao atingir os seios, atinge também a identificação mais visível do "ser mulher", uma vez que os seios têm forte representação com a identidade feminina e com o funcionamento da personalidade das mulheres (Conde, Lemos, Pozati \& Ferreira, 2016)

Há ainda as questões femininas relativas à sensualidade e à sexualidade, que trazem profundos questionamentos físicos e emocionais e podem abalar intrinsecamente a estrutura psíquica de uma mulher e, consequentemente sua autoestima, gerando pensamentos angustiantes diante de um diagnóstico positivo para câncer de mama (Salci, \& Marcon, 2008; Salci \& Marcon, 2011).

Além dos efeitos negativos que o câncer provoca, como baixa-estima, depressão entre outros, os tratamentos e procedimentos trazem efeitos colaterais que também precisam ser considerados. Como exemplo, a queda dos cabelos, que é destruído por causa da "doença" (Menezes, Sculz, \& Peres, 2012).

A paciente precisa contar com alguma fonte de apoio seja no âmbito familiar, com amigos, no trabalho e s profissionais da saúde. Ou ainda exercitando alguma atividade que possa recuperar sua fonte de autoestima, prazer, vitalidade ou esperança. Neste cenário encontram-se inúmeras pesquisas que tem apontado o impacto da espiritualidade/religiosidade como fontes de amparo psíquico e emocional em situações de adversidades (Soratto, da Silva, Zugno, \& Daniel, 2016; Botelho, \& Pereira, 2015; Conde, Lemos, Pozati, \& Ferreira, 2016).

A espiritualidade pode dar uma nova perspectiva sobre a enfermidade, transformando a forma como se a vivencia e percebe o problema, proporcionando um maior alivio da dor e da angústia apresentadas no processo de adoecimento. Tanto a espiritualidade como a religiosidade permitem à pessoa se apegar à uma "força-maior", ajudando-a em uma fase difícil (Cardoso, 2013; Oliva, Faria, Martins, Figueiredo, Moura, Zanatta, Wong $\&$ Pereira, 2015).

As questões sobre a espiritualidade e a repercussão desta na saúde são importantes para a adesão ao tratamento, para a busca da qualidade de vida e para o bem-estar. A tentativa de um equilíbrio emocional e espiritual pode refletir no corpo, com sensações de bem-estar, tranquilidade e paz; situações essas, que são essenciais para a qualidade de vida e saúde (Araújo, Guerra, Da Silva, Martins, Braga \& Almeida, 2015).

A relação entre espiritualidade e saúde pode oferecer às pessoas um maior conforto no enfrentamento da doença e auxiliá-las a lidar com o alto nível de estresse e adaptações que o adoecer ocasiona (Areco, 2016).

Estudos têm demonstrado que a esta tem trazido diversos benefícios no âmbito da saúde, como uma participação maior na realização do tratamento, esperança de que possa ter êxito na cura (incluindo a redução de sintomas depressivos), formação de significado da vida, apoio social, entre outros (Hoffmann, Müller \& Rubin, 2006; Gobatto, \& Araujo, 2010; Reginato, Benedetto, Gallian, 2016; Gobatto, \& Araujo, 2013; ).

Este estudo propõe investigar qual a importância da espiritualidade e da religiosidade no processo de enfrentamento (coping) da doença para um grupo de pacientes mastectomizadas. Buscou também conhecer se a prática religiosa era um hábito anterior ao adoecimento ou se aflorou após a constatação da enfermidade.

\section{Metodologia}

Trata-se de um estudo qualitativo que utilizou como instrumento a entrevista aberta com roteiro de perguntas elaborado pelas pesquisadoras. A metodologia qualitativa no âmbito da saúde tem por intuito compreender o significado individual ou coletivo que determinado fenômeno, como o câncer de mama, tem para a vida das pessoas, deixando de estudar o fenômeno em si. Será investigado o que o evento ocasiona na vida do indivíduo (Turato, 2005).

Participaram da pesquisa cinco mulheres que tiveram os seios parcial ou totalmente retirados em função do câncer de mama (mastectomia). Todas participavam de um grupo de reabilitação na clínica de fisioterapia de uma universidade privada no interior de São Paulo e foram encaminhadas pela rede pública de saúde. Elas tinham dois atendimentos semanais em que eram realizados atendimentos individuais e em grupo. $\mathrm{O}$ atendimento individual tinha por objetivo reabilitar os 
movimentos dos braços e dos músculos que se localizam próximo aos seios que passaram por redução/retirada e a reeducação postural. No atendimento grupal, elas realizavam exercícios físicos visando o reestabelecimento da postura, uma vez que a retirada da mama resulta em alterações posturais importantes. Também recebiam orientações sobre saúde em geral, nutrição e, quando solicitado por elas, ou identificada a necessidade de orientação psicológica, elas eram encaminhadas ao setor de Psicologia da universidade.

A coleta de dados ocorreu no mês de agosto de 2015, durante este período oito mulheres estavam em atendimento na clínica, entretanto apenas cinco concordaram em participar desse estudo. O convite foi feito durante o encontro grupal.

Das cinco participantes do estudo, somente três autorizaram que as entrevistas fossem gravadas. As demais foram entrevistadas e tomou-se nota das respostas manualmente. Todas as entrevistas ocorreram em sala reservada da clínica, em dia e data previamente agendados, após a leitura do Termo de Consentimento Livre e Esclarecido.

As entrevistas foram posteriormente transcritas, após a leitura exaustiva de todas as respostas, gravadas ou não. Em seguida foram agrupadas as respostas que tinham o mesmo sentido interpretativo, e sobre os temas propostos construíram-se categorias, conforme orienta a análise do conteúdo na modalidade temática, que considera além do que foi dito nas entrevistas as observações do pesquisador (Silva e Fossa, 2015).

O estudo foi apresentado e aprovado pelo Comitê de Ética em Pesquisa sob No 25477413.0.0000.5495, conforme prevê a Resolução 466/12.

\section{Resultados e Discussão}

Para a caracterização das participantes, foram considerados a idade, escolaridade, religião, e o tipo de tratamento que haviam recebido.

As falas das pacientes foram originalmente mantidas. Foram formadas duas categorias temáticas, a partir da seleção de palavras e sinônimos que melhor expressavam o sentido das perguntas.

\section{1 - Reação Inicial ao Diagnóstico e Enfrentamento da Enfermidade}

Para a formação da primeira categoria utilizou-se as perguntas "Qual foi sua reação inicial ao diagnóstico?" e "De onde ou como tem encontrando força para superar a enfermidade?"

Com estas perguntas objetivou-se compreender como as participantes reagiram ao diagnóstico de câncer de mama, de que forma o enfrentaram e quais foram os apoios por elas percebidos que foram importantes para o enfrentamento do diagnóstico, bem como a aceitação da enfermidade, do tratamento e suas consequências.

Quadro 1. Caracterização das participantes

\begin{tabular}{|c|c|c|c|c|}
\hline Participantes & Idade & Escolaridade & Religião & Tratamento atual \\
\hline P1 & 68 anos & Fundamental incompleto & Católica & Fisioterapêutico. \\
\hline P2 & 62 anos & Fundamental incompleto & Adventista & $\begin{array}{l}\text { Fisioterapêutico } \\
\text { Avaliação da mama. }\end{array}$ \\
\hline P3 & 48 anos & Superior Completo & Espírita & $\begin{array}{l}\text { Fisioterapêutico Avaliação } \\
\text { da mama. }\end{array}$ \\
\hline P4 & 79 anos & Fundamental incompleto & Católica & $\begin{array}{l}\text { Fisioterapêutico } \\
\text { Avaliação da mama }\end{array}$ \\
\hline P5 & 57 anos & Média Completo & Católica & $\begin{array}{l}\text { Fisioterapêutico } \\
\text { Quimioterapia e } \\
\text { Radioterapia }\end{array}$ \\
\hline
\end{tabular}


As participantes tiveram reações diversas, algumas disseram que enfrentaram o diagnóstico de forma tranquila, sem dar tanta importância ao mesmo enquanto que outras relataram medo, susto e até raiva ao se depararem com o diagnóstico.

"Levei na brincadeira, sem medo" (P4,79 anos).

"Não desesperei, disse que morreria com peito ou sem peito [...]" (P2, 62 anos).

"Meu mundo caiu.... fiquei sem voz, não acreditava. Um misto de raiva, impotência e desânimo. Só pensei o pior" (P1. 68 anos).

"Estranho, não estava esperando, estava sozinha, não acreditava, fiquei sem chão, ficou tudo perdido. Foi horrível a sensação” (P5, 57 anos).

"Dá muito medo, levei muito susto, é aquela velha história, você acha que vai acontecer com todo mundo menos com você." ( P3, 48 anos).

Em estudo realizado por Guerrero, Zago, Sawada e Pinto (2011), com pacientes oncológicos de ambos os sexos, alguns participantes tiveram reações semelhantes às descritas neste estudo: sentimentos de desespero, perplexidade, tristeza e indignação para uns, enquanto em outros relatos as reações foram mais serenas com demonstração de confiança no tratamento e prognóstico.

Durante todo o processo da doença, desde o diagnóstico é comum a vivencia de sentimentos de medo, sofrimento e ansiedade, incluindo o temor da retirada da mama, o preconceito da sociedade e do parceiro, temor da morte incluindo sentimentos depressivos podendo ocorrer autodepreciação, sentimento de impotência e frustração sobre algo que fugiu ao controle. Com a cirurgia de retirada do câncer e seu tratamento ocorrem mudanças cotidianas e interrupções de hábitos rotineiros na vida da mulher. Além disso, a doença altera a vida da mulher, fazendo com esta perca papeis sociais e ocupacionais, no âmbito profissional, familiar e com sua sexualidade (Majewski, Lopes, Davoglio \& Leite, 2012; (Faria, Fangel, de Almeida, Prado \& do Prado, 2016).

Quando responderam sobre quais os elementos foram importantes para o enfrentamento da enfermidade destacou-se o papel da espiritualidade, de Deus e da religião.

"Pra mim Deus é tudo. E entreguei minha vida nas mãos de Maria, nossa Mãe Santissima que sabe o que é a dor (P1). "

"Oro sempre, assim como sempre fir. Entrego tudo para Deus e confio. A gente tem mania de orar, entregar para Deus e pedir para fazer a vontade dele, mas na nossa cabeca a gente quer que

\author{
ele faça a nossa” (P2). \\ "Através da espiritualidade [...]” (P3). \\ “Deus e a religião” (P4). \\ "É Deus, lá que vem a força. É na comunhão que recebo na \\ santa missa (P5) “
}

A espiritualidade muitas vezes atua na forma com a qual as pessoas enfrentam determinadas adversidades, aumentando a e resiliência e auxiliando no enfrentamento individual de cada um frente à doença. A fé e a espiritualidade são apoios no enfrentamento do diagnóstico do câncer, em seu tratamento e também atuam como consolo frente a possibilidade da morte (Soratto, da Silva, Zugno \& Daniel, 2016).

A espiritualidade surge como expressão da identidade e de sentido da vida individual, construído a partir da própria história, experiências e anseios. Nesse sentido, a fé e a busca da espiritualidade, tem o poder de aliviar o sofrimento do paciente, incluindo o sofrimento dos familiares, permitindo uma mudança de perspectiva sobre o evento do adoecimento (Aquino \& Zago, 2007).

Outras fontes de apoio são os familiares, como comenta a participante P1, sobre a importância do apoio familiar, para sua recuperação e enfrentamento da doença, conforme disse ter encontrado forças na (...) fé em Deus e ajuda dos filhos e do marido (P1).

É sabido da importância do apoio da família para a recuperação do paciente com câncer, vários estudos têm demonstrado a relevância do papel dos familiares para o enfrentamento da doença e adesão ao tratamento, que é desgastante, muitas vezes doloroso e que deixa o paciente abatido tanto físico, como emocionalmente. A participação da família no tratamento do câncer de mama e na recuperação da paciente mastectomizadas é importantíssima, visto que, ela é um dos principais apoios que a paciente encontra para lidar com o estresse associado ao tratamento, como a retirada dos seios, bem como os problemas de autoimagem e com a sexualidade (Tavares \& Trad, 2010; Conde, Lemos, Pozati \& Ferreira, 2016).

\section{Espiritualidade como Recurso no Enfrentamento da Doença}

Com as perguntas "Que papel tem ou teve a espiritualidade/ religião, neste processo?”, e "A questão espiritual já era parte de sua rotina on despertou durante esse processo?", buscou-se compreender a dinâmica da espiritualidade como recurso de enfrentamento do câncer de mama, e também saber se a espiritualidade aflorou durante o tratamento ou na confirmação do diagnóstico. 
“É muito importante porque sem Deus não daria conta. Deus é o nosso tudo, é preciso sempre pedir proteção para Deus" (P1).

"Sempre oro e confio em Deus, entrego nas mãos dele. Se não orasse não confiaria [...]” (P2)

“Ah! É muito importante, muito importante mesmo. (...). Deus faz $80 \%$ se não for $90 \%$ e o que sobra pra gente fazer de vez enquanto a gente ainda escorrega, porque você tem momentos de baixo astral, mas eu acredito assim, foi o que me sustentou mesmo, com certeza “(P3). " Toda a importância do mundo. Me deu coragem, força para continuar e aceitar minha condição, o tratamento e também esperança de que me curaria. Não fosse isso, não sei onde ia parar (P4).”

" Eu sempre fui de frequentar a igreja, desde menina, sempre gostei da missa. Para mim, tomar a comunhão é parte importante para minha vida, e quando soube que estava doente, minha fé no sangue e no poder do Cristo, só aumentou (P5). “

Em estudo semelhante, realizado com pacientes oncológicos, Fornazari e Ferreira (2010) encontraram em seus relatos situações de enfrentamento do problema a partir de uma referência à existência de um deus.

A religião e a espiritualidade proporcionam ao paciente mudança no enfrentamento da doença, modificando a forma como o paciente e as pessoas próximas a este, visualizam o problema, gerando alivio da dor, estresse e temor (Cardoso, 2013).

A junção entre as dimensões espirituais e religiosas no tratamento da enfermidade exige dos profissionais envolvidos uma visão ética, com um grau elevado de conhecimento sobre a importância das crenças e valores de cada paciente, para que assim possa-se utilizar de forma benéfica dentro do processo de tratamento/cura (Puggina \& Silva, 2015).

Sobre saber se a espiritualidade já era parte da rotina destas mulheres, as participantes P1, P2 e P4 disseram que já tinham uma referência espiritual e prática religiosas anteriores ao diagnóstico. Todas elas disseram que tinham uma vivência espiritual antes mesmo da doença, apontando a relação desta com a prática de suas religiões, entretanto as participantes P3 e P5 confessaram que a intensidade com a qual passaram a vivenciar sua espiritualidade, aumentou após o diagnóstico de câncer.

“Já fazia parte, mas não com tanta intensidade [...]" (P3). “Já era, mas agora despertou mais" (P5).
A religião é um fenômeno que aponta a consciência que o ser humano tem de suas limitações e da finitude da vida, mas mantém o desejo de superar tal realidade, buscando na religião, respostas para questionamentos e um sentido para a sua existência (Melo, Sampaio, Souza, \& Pinto, 2015).

Para Soratto, da Silva, Zugno e Daniel (2016) a espiritualidade surge como força para o enfrentamento da doença e de seu tratamento. Quando o paciente está sem sentido ou forças para lutar pela vida, a religião e a fé são primordiais, visto que, o processo de adoecimento traz muita dor, sofrimento e inseguranças sobre o futuro, tanto para o paciente quanto para os familiares envolvidos. Nesse sentido, a espiritualidade e religião, proporcionam efeitos benéficos para a qualidade de vida da paciente.

No encerramento das entrevistas era deixado um momento para que elas pudessem se expressarem livremente. Muitas utilizaram este momento para reforçar suas convicções na fé em Deus e outras ressaltaram também a importância da escuta para se sentirem ajudadas:

"O que posso te falar? Que a gente precisa sempre manter o espírito da gente em Deus [...]” (P1).

" Digo que é importante crer em uma força superior, Deus, sabe? Mas também é muito importante a gente se sentir protegida, amparada pelas pessoas, sermos ouvidas. O medo muitas vezes toma conta, mesmo a gente tendo fé, e alguém para nos ouvir e apoiar e até lembrar do poder de Deus, é importantíssimo" (P2).

"Comecei a olhar muito, para as pessoas sozinhas, sabe? Para olhar para as pessoas que não tem câncer, mas têm outros tipos de doenças e de problemas, muito maiores talvez do que um câncer, pessoas muito jovens com doenças psicológicas, esquizofrenia, pessoas de idade com Parkson, Alzheimer, que não tem ninguém por elas, e eu comecei a ficar, mais atenta às emoções das pessoas diante das dificuldades. (...). Então são coisas assim, depende muito do que a pessoa quer conversar também. Tem gente que se fecha, mas pelo menos é alguém tipo apoio, que qualquer um nessa hora é válido" (P3).

"De tudo que foi dito creio no poder de Deus, da oração, da ajuda das pessoas e dos profissionais que trabalham e atende a gente. Sem eles para ouvir e orientar também é difícil" (P4).

"Às vezes sinto vontade de conversar com meu marido" (P5). 
A importância de integrar na dimensão do trabalho clínico a espiritualidade e a religião é apontada por Stroppa e Moreira-Almeida (2008). Os autores ainda comentam que a "religião mobiliza alguns dos mais profundos e apaixonados sentimentos humanos, e não se constitui surpresa o fato de influenciarem a saúde", salientando ainda sobre as mudanças de comportamento que são derivadas da religiosidade e que isso pode fazer com que sejam desestimulados hábitos que prejudiquem a saúde e consequentemente venha fazer bem não só à saúde física, mas psicológica também (Stroppa \& Moreira-Almeida, 2008, p.9).

A referência a uma dimensão espiritual/religiosa, por parte dos pacientes, como algo que lhes auxilia no enfrentamento da enfermidade e lhes concede força interior $\mathrm{e}$ coragem para lidar com a situação, deve, portanto, ser compreendida pelos profissionais de saúde e integrada em suas práticas e não entendido como um recurso que não cabe na relação que se estabelece entre este e o paciente (Cardoso, 2013).

Há de se considerar, portanto, uma prática que enseje a espiritualidade como parte do processo da existência humana, suas emoções e a forma com que o sujeito se relaciona e vive sua própria vida. Neste sentido as psicoterapias baseadas na linha transpessoal podem ter eficácia para os pacientes que buscam as relações entre o que ocorre com sua saúde e seu propósito de vida, incluindo intervenções espirituais/ religiosas em suas práticas (Veit \& Castro, 2013).

As evidências da importância da espiritualidade como enfrentamento das doenças orientaram este trabalho, confirmando o que igualmente já se encontra na literatura, cujos estudos vêm sendo realizados sobre a dimensão e a importância da espiritualidade no tratamento e no enfrentamento de enfermidades.

\section{Considerações finais}

As participantes demonstraram que no percurso de seus tratamentos contaram principalmente com o apoio que a espiritualidade pode proporcionar a cada uma, inclusive dentro de suas religiões. Elas atribuíram um papel importante à espiritualidade, ao dizerem que esta lhes renovou as forças para a luta diária contra a doença, dando-lhes um propósito para viver, contribuindo, portanto, tanto para o enfrentamento do diagnóstico e tratamento, como para seus processos de recuperação.

Embora não tenha sido objetivo do estudo discutir sobre a atuação do profissional de saúde, pensa-se na importância de sua escuta para a compreensão da dimensão da espiritualidade como importante recurso de enfrentamento e recuperação da enfermidade, o que implica na necessidade de rever a formação acadêmica e profissional dos futuros profissionais da saúde. Se por muito tempo essa discussão ficou relegada ao plano teológico e filosófico, atualmente as ciências têm se ocupado de entender a dimensão da espiritualidade na prática clínica e sua importância no enfrentamento das doenças, bem como na aceitação do diagnóstico e tratamento.

Para essas participantes foi importante poder contar um pouco de suas histórias, talvez as relatando pela primeira vez, falar sobre seus sentimentos diante da situação da enfermidade e da realidade da mastectomia.

A experiência de partilhar o quanto que a fé e a espiritualidade foram imprescindíveis para a recuperação, durante o processo de adoecimento, mostra-nos a importância de mais estudos e pesquisas nessa área, envolvendo a interface espiritualidade e saúde.

\section{Referências}

Aquino V.V.\& Zago M.M.F. (2007) O significado das crenças religiosas para em grupo de pacientes oncológicos em reabilitação. Rev Latino-am Enfermagem ;15(1), 42-47 http://www.scielo.br/scielo.php?script=sci_ nlinks\&ref=000164\&pid=S0034-7167201100010000800013\&lng=en.

Araújo, M. Â. M., Guerra, D. R., da Silva, A. L., Martins, L. G. F., Braga, V. A. B., \& de Almeida, A. N. S. (2015). Os Conceitos de Sentido da Vida: reveladores da espiritualidade da pessoa com câncer. Revista Logos \& Existência: Revista da Associação Brasileira de Logoterapia e Análise Existencial, 4(2). http://periodicos.ufpb.br/index.php/le/article/view/21726

Areco, F. D. S. (2016). A espiritualidade para mulheres com câncer de mama: uma revisão integrativa (Doctoral dissertation, Universidade de São Paulo). Escola de enfermagem de Ribeirão Preto, Universidade de São Paulo, Ribeirão Preto. http://www.tese.usp.br/teses/disponiveis/22/22133/tde16012017-171409/en.php

Botelho, A. S. C., \& Pereira, M. da G. (2015). Qualidade de vida, otimismo, enfrentamento, morbidade psicológica e estresse familiar em pacientes com câncer colorrectal em quimioterapia. Estudos de Psicologia (Natal), 20(1), 50 60. https://dx.doi.org/10.5935/1678-4669.20150007

Cardoso, A. H. A. (2013). Espiritualidade e câncer em pacientes submetidos a tratamento quimioterápico. Trabalho de conclusão de curso - Departamento de Enfermagem da Universidade Estadual da Paraíba, Centro de Ciências Biológicas e da Saúde, Campina Grande. http://dspace.bc.uepb.edu.br/ jspui/bitstream/123456789/5222/1/PDF\%20-\%20Agna\%20Hanna\%20 Alencar\%20Cardoso.pdf

Conde, C., Lemos, T., Pozati, M., \& Ferreira, M. (2016). A repercussão do diagnóstico e tratamento do câncer de mama no contexto familiar. CIAIQ2016, 2. http://proceedings.ciaiq.org/index.php/ciaiq2016/ article/view/913

Faria, N. C., Fangel, L. M. V., de Almeida, A. M., Prado, M. A. S., \& do Prado De, M. M. R. (2016). Ajustamento psicossocial após mastectomia-um olhar sobre a qualidade de vida. Psicologia, Saúde e Doenças, 17(2), 201-213. http:// www.redalyc.org/pdf/362/36248047007.pdf

Fornazari, S.A; \& Ferreira, R.E.R. (2010) Religiosidade/Espiritualidade em Pacientes Oncológicos: Qualidade de Vida e Saúde. Psicologia: Teoria e Pesquisa. Universidade Estadual de Londrina. 26(2),265-272. < http://www.scielo. $\mathrm{br} / \mathrm{pdf} / \mathrm{ptp} / \mathrm{v} 26 \mathrm{n} 2 / \mathrm{a} 08 \mathrm{v} 26 \mathrm{n} 2>$.

Gobatto, C. A., \& Araujo, T.C.C. F.de. (2010). Coping religioso-espiritual: reflexões e perspectivas para a atuação do psicólogo em oncologia. Revista da SBPH, 13(1), 52-63. http://pepsic.bvsalud.org/scielo.php?script=sci_ arttext\&pid=S1516-08582010000100005\&lng=pt\&tlng=pt. 
Gobatto, C., \& Araujo, T. (2013). Religiosidade e espiritualidade em oncologia: concepções de profissionais da saúde. Psicologia USP, 24(1), 11-34. doi: http://dx.doi.org/10.1590/S0103-65642013000100002.

Guerrero, G. P., Zago, M. M. F., Sawada, N. O., \& Pinto, M. H. (2011). Relação entre espiritualidade e câncer: perspectiva do paciente. Revista Brasileira de Enfermagem, 64(1), 53-59. http://www.producao.usp.br/handle/BDPI/3494

Hoffmann, F.S., Müller, M.C.,\& Rubin, R. (2006). A mulher com câncer de mama: apoio social e espiritualidade. Mudanças - Psicologia da Saúde, 14 (2), jul-dez.

INCA. (2017) Instituto Nacional de Cancêr José Alencar Gomes da Silva. Tipos de Cancêr: Mama. http://www2.inca.gov.br/wps/wcm/connect/tiposdecancer/ site/bome/mama

Majewski, J. M., Lopes, A. D. F., Davoglio, T., \& Leite, J. C. D. C. (2012). Qualidade de vida em mulheres submetidas à mastectomia comparada com aquelas que se submeteram à cirurgia conservadora: uma revisão de literatura. Ciência \& Saúde Coletiva, 17(3), 707-716. http://www.scielosp. org/pdf/csc/v17n3/v17n3a17.pdf

Melo, C. D. F., Sampaio, I. S., Souza, D. L. D. A., \& Pinto, N. D. S. (2015). Correlação entre religiosidade, espiritualidade e qualidade de vida: uma revisão de literatura. Estudos e Pesquisas em Psicologia, 15(2), 447-464. http://pepsic. bvsalud.org/scielo.php?script=sci_arttext\&pid=S1808-42812015000200002

Menezes, N. N. T. de, Schulz, V. L., \& Peres, R. S. (2012). Impacto psicológico do diagnóstico do câncer de mama: um estudo a partir dos relatos de pacientes em um grupo de apoio. Estudos de Psicologia (Natal), 17(2), 233240. https://dx.doi.org/10.1590/S1413-294X2012000200006

Oliva, L. F. D. G., Faria, C. C., Martins, C. V., Figueiredo, M. G., de Moura Zanatta, R., Wong, T. G., \& Pereira, A. C. A. (2015). Impactos psicossociais do diagnóstico e tratamento em pacientes com câncer de mama em Hospital Oncológico Campo Grande -MS. Ensaios e Ciência: C. Biológicas, Agrárias e da Saúde, 17(4). http://pgsskroton.com.br/seer/index.php/ensaioeciencia/ article/view/2320

Puggina, A. C. G., \& da Silva, M. J. P. (2015). Religião/espiritualidade como estratégia de enfrentamento de familiares de pacientes com desordem de consciência. Revista Saúde-UNG, 9(3-4), 05-17. http://revistas.ung.br/index. $\mathrm{php/saude/article/view/2136}$
Reginato, V., Benedetto, M. A. C. de, \& Gallian, D. M. C. (2016). Espiritualidade e saúde: uma experiência na graduação em medicina e enfermagem. Trabalho, Educação e Saúde, 14(1), 237-255. https://dx.doi.org/10.1590/19817746-sip00100.

Salci, M. A., \& Marcon, S. S. (2008). De cuidadora a cuidada: quando a mulher vivencia o câncer. Texto Contexto Enferm, 17(3), 544-51. https:// www.researchgate.net/profile/Sonia_Marcon/publication/262775917_ From_caretakers_to_taken_care_of_when_women_face_cancer/ links/5543cebf0cf24107d39636ce.pdf

Salci, M. A., \& Marcon, S. S. (2011). Enfrentamento do câncer em família. Texto and Contexto Enfermagem, 20, 178. http://www.index-f.com/ textocontexto/2011pdf/20s-178.pdf

Silva, A.H., \& Fossa, M. I. T (2015). Análise de conteúdo: exemplo de aplicação da técnica para análise de dados qualitativos. Qualitas Revista Eletrônica, 16(1) http://revista.uepb.edu.br/index.php/qualitas/article/view/2113

Soratto, M. T., da Silva, D. M., Zugno, P. I., \& Daniel, R. (2016). Espiritualidade e Resiliência em Pacientes Oncológicos. Saúde e Pesquisa, 9(1), 53-63. http://periodicos.unicesumar.edu.br/index.php/saudpesq/article/view/4284

Stroppa, A.; Moreira-Almeida, A.(2008). Religiosidade e Saúde. In M I Salgado \& G. Freire (Orgs), Saúde e Espiritualidade: uma nova visão da medicina (Cap. 20, pp. 427-443). Belo Horizonte: Inede.< http://www.scielo.br/ scielo.php? script=sci_arttext\&pid=S0101-60832009000500003 $>$.

Tavares, J. S. C. \& Trad, L. A. B. (2010). Estratégias de enfrentamento do câncer de mama: um estudo de caso com famílias de mulheres mastectomizadas. http://www.repositorio.ufba.br/ri/handle/ri/2358

Turato, E. (2005). Qualitative and quantitative methods in health: definitions, differences and research subjects .Revista de Saúde Pública, 39(3), 507-514 doi: http://dx.doi.org/10.1590/S0034-89102005000300025

Veit, C. M. \& Castro, E. K. (2013). Coping religioso/espiritual e câncer de mama: Uma revisão sistemática da literatura. Psicologia, Saúde \& Doenças, 14(1), 1-22. http://www.scielo.mec.pt/scielo.php?script=sci_ arttext\&pid $=$ S1645-00862013000100001\&lng=pt\&tlng=pt.

Submetido em: 2-10-2017

Aceito em: 7-1-2019 